\title{
Diabetes Mellitus in Older Adults: Underdiagnosis and Undertreatment
}

$\mathrm{I}$ you are interested in type 2 diabetes mellitus, one of the leading causes of increased morbidity and mortality in older adults, this is the best of times and the worst of times. First, the good news. A growing body of evidence provides a stronger framework for understanding the scope of this condition, its impact on the lives of older people, and the potential for substantially improved therapeutic outcomes. The 1997 report of the American Diabetes Association's Expert Committee $^{1}$ summarizes some of these advances, proposes new diagnostic criteria that have been widely adopted (but see more about this below), and recommends screening for diabetes on a regular basis in all older adults. Intervention studies such as the United Kingdom Prospective Diabetes Study and various lipid-lowering trials have demonstrated that rates of both the microvascular and macrovascular adverse outcomes of type 2 diabetes can be reduced, ${ }^{2-4}$ although much of this data requires extrapolation from middle-aged to older-aged populations. Thus, people with diabetes mellitus may particularly benefit from aggressive identification and treatment of hypertension and lipid disorders. A diagnosis of diabetes mellitus puts an individual in the highest risk group in the current classification from the NIH Joint National Committee on High Blood Pressure and sets an aggressive target of less than 130/85 for blood pressure control in such individuals. ${ }^{5}$ Based on their high risk for cardiovascular disease, ${ }^{6}$ lowering the target LDL cholesterol goal for people with a diagnosis of diabetes mellitus to less than $100 \mathrm{mg} / \mathrm{dL}$, regardless of the presence of documented coronary heart disease, is being advocated and may be adopted as an official recommendation.

The impact of glucose lowering on outcomes is still somewhat controversial, although there now seems little doubt that aggressive lowering of glucose levels will reduce the rate of microvascular complications of diabetes mellitus. Interventions for specific diabetes complications such as retinopathy and nephropathy have demonstrated efficacy. A growing list of highly effective therapeutic agents are available to treat hypertension and hyperlipidemia in people with diabetes. Furthermore there is now a rapidly growing list of options for treatment of hyperglycemia with agents available to address a variety of contributory mechanisms such as impaired insulin secretion, increased hepatic glucose production, and impaired insulin-mediated glucose uptake, as well as interfering with gastrointestinal absorption of glucose. The opportunities for combination therapy, given different modes of action of various agents, are expanding greatly.

Although this could be the best of times for diagnosis and treatment of diabetes mellitus in older adults, many serious challenges confront us. The new ADA diagnostic criteria focus on fasting serum glucose, a relatively easy test to use for screening, and downplay the use of glucose tolerance testing. The ADA's Expert Committee recognized that focusing on the fasting serum glucose will lead to underdiagnosis, missing individuals who have marked hyperglycemia after glucose challenge, so called isolated postchallenge hyperglycemia. However, they may not have realized that isolated postchallenge hyperglycemia is particularly common among older adults. ${ }^{7,8}$ Thus, the new ADA criteria and focus on the fasting glucose level will lead to underdiagnosis of type 2 diabetes mellitus particularly in older adult populations. Unfortunately, older individuals with isolated postchallenge hyperglycemia seem to have an increased risk for cardiovascular disease equal to those with overt fasting hyperglycemia..$^{9-11}$ However, this is only part of the problem of underdiagnosis. Epidemiologic studies suggest that 30 to $50 \%$ of people who meet criteria for diabetes mellitus in the United States (even using conservative 1997 ADA criteria), do not know that they have diabetes. ${ }^{7}$ This is not surprising as there are also many older people with undiagnosed hypertension, hyperlipidemia, and other disorders. Even worse, despite the many advances in therapeutics, the overall population success in treatment of these important risk factors has been disappointing. Targets for hypertension, hyperlipidemia, and hyperglycemia are often not achieved. $2,5,12$ The paper by Shorr et al. in this issue of the Journal of the American Geriatrics Society ${ }^{13}$ confirms and extends previous studies reporting lack of adequate control of hyperglycemia in many older adults. ${ }^{14,15}$ We can take little solace in the finding that the percentage of older people with poor control may be slightly less than in other age groups, although this is an interesting finding that merits further investigation. It could represent a survival effect, with disproportionate early death of those with the poorest diabetes control, or it could possibly reflect a somewhat earlier diagnosis or responsiveness to treatment of some older people.

\section{See also p 264}

The causes of the troublesome gap between what we know and what we are able to deliver successfully to patients are uncertain and are likely multifactorial. It is hard to believe that lack of physician knowledge is important given the barrage of publicity and educational efforts addressing management and targets for hypertension, hyperlipidemia, and diabetes mellitus. But can a physician adequately present information to patients and provide needed therapeutic adjustments in a 15-minute office visit? Undoubtedly, we need to come up with better and more efficient ways of providing education and follow-up to patients so that there is greater motivation for adherence to sometimes complex diabetes 
interventions. ${ }^{16}$ For a patient with diabetes alone, in addition to a diet prescription and exercise recommendation, therapeutic guidelines might lead to multiple drug regimens for hyperglycemia and blood pressure control as well as an additional drug for hyperlipidemia. Other drugs would be indicated for individuals with evidence of diabetes complications such as nephropathy, existing coronary disease, etc. Tasks often presented as simple, such as use of a blood glucose monitor, are, in fact, very complex. ${ }^{17}$ Surely the dramatic advances occurring in information technology can help us deal with this complexity and do a better job of translating the growing body of medical knowledge into improved outcomes for our older patients with type 2 diabetes mellitus.

Jeffrey $B$. Halter, $M D$

Director, Geriatrics Center, University of Michigan, and VA Ann Arbor Health Care System Ann Arbor, Michigan

\section{REFERENCES}

1. The Expert Committee on the Diagnosis and Classification of Diabetes Mellitus. Report of the expert committee on the diagnosis and classification of diabetes mellitus. Diabetes Care 1997;20;1183-1197.

2. UK Prospective Diabetes Study (UKPDS) Group. Intensive blood-glucose control with sulphonylureas or insulin compared with conventional treatment and risk of complications in patients with type 2 diabetes (UKPDS 33). Lancet 1998;352:837-853.

3. UK Prospective Diabetes Study Group. Tight blood pressure control and risk of macrovascular and microvascular complications in type 2 diabetes. BMJ 1998;317:703-13.

4. The Long-Term Intervention with Pravastatin in Ischaemic Disease (LIPID) Study Group. Prevention of cardiovascular events and death with pravastatin in patients with coronary heart disease and a broad range of initial cholesterol levels. N Engl J Med, 1998;339:1349-1357.

5. National Institutes of Health, National High Blood Pressure Education Program. The Sixth Report of the Joint National Committee on Prevention, De- tection, Evaluation, and Treatment of High Blood Pressure. Bethesda, MD: US Department of Health and Human Services, National Heart, Lung, and Blood Institute. 1997. NIH No. 98-4080.

6. Haffner SM, Lehto $S$, Ronnemaa $T$ et al. Mortality from coronary heart disease in subjects with type 2 diabetes and in nondiabetic subjects with and without prior myocardial infarction. N Engl J Med, 1998;339:229-234.

7. Harris MI, Flegal KM, Cowie CC et al. Prevalence of diabetes, impaired fasting glucose, and impaired glucose tolerance in U.S. adults. The Third $\mathrm{Na}$ tional Health and Nutrition Fxamination Survey, 1988-1994. Diabetes Care $1998 ; 21: 518-524$.

8. Barrett-Connor E, Ferrara A. Isolated postchallenge hyperglycemia and the risk of fatal cardiovascular disease in older women and men. Diabetes Care 1998;21:1236-1239.

9. Barzilay J, Spiekerman CF, Wahl PW et al. Cardiovascular disease in older adults with glucose disorders: Comparison of American Diabetes Association criteria for diabetes mellitus with WHO criteria. Lancet 1999;354:622-625.

10. The DECODE study group on behalf of the European Diabetes Epidemiology Group. Glucose tolerance and mortality: Comparison of WHO and American Diabetes Association diagnostic criteria. Lancet 1999;354:617621.

11. Rodriguez BL, Lau N, Burchfiel CM et al. Glucose intolerance and 23-year risk of coronary heart disease and total mortality. Diabetes Care 1999;22: 1262-1265.

12. Schrott HG, Bittner V, Vittinghoff $E$ et al. for the HERS Research Group. Adherence to National Cholesterol Education Program treatment goals in postmenopausal women with heart disease: The Heart and Estrogen/Progestin Replacement Study (HERS). JAMA 1997;277:1281-1286.

13. Shorr RI, Franse LV, Resnick HE et al. Glycemic control of older adults in the US with type 2 diabetes: Findings from the Third National Health and Nutrition Examination Survey, 1988-1994. J Am Geriatr Soc 2000;48:264267.

14. Weiner JP, Parente ST, Garnick DW et al. Variation in office-based quality. A claims-based profile of care provided to medicare patients with diabetes. JAMA 1995;273:1503-1508.

15. Kell SH, Drass J, Bausell RB et al. Measures of disease control in Medicare beneficiaries with diabetes mellirus. J Am Geriatr Soc 1999;47:417-422.

16. Glasgow RE, Wagner EH, Kaplan RM et al. If diabetes is a public health problem, why not treat it as one? A population-based approach to chronic illness. Ann Behav Med 1999;21:1-13.

17. Rogers WA, Mykityshyn AL, Campbell RH, Fisk AD. Only three easy steps? User-centered analysis of a simple medical device (AAA-TR-9902). Atlanta, GA: Georgia Institute of Technology School of Psychology, Attention, Abilities, and Aging Laboratory, 1999. 